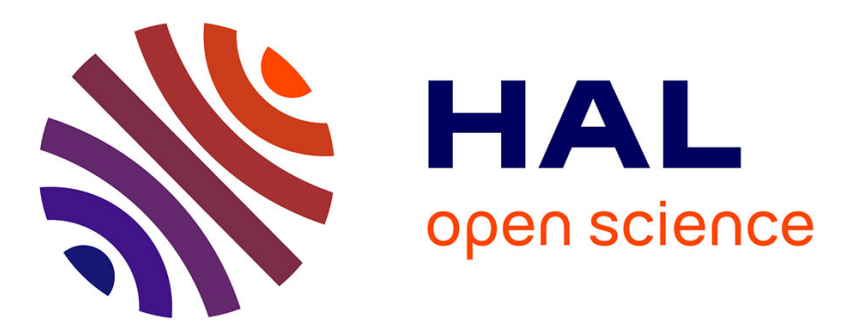

\title{
Dynamic holographic memory showing readout, refreshing, and updating capabilities
}

Sylvain Boj, Gilles Pauliat, Gérald Roosen

\section{To cite this version:}

Sylvain Boj, Gilles Pauliat, Gérald Roosen. Dynamic holographic memory showing readout, refreshing, and updating capabilities. Optics Letters, 1992, 17 (6), pp.438-440. hal-00856163

\section{HAL Id: hal-00856163 \\ https://hal-iogs.archives-ouvertes.fr/hal-00856163}

Submitted on 2 Sep 2013

HAL is a multi-disciplinary open access archive for the deposit and dissemination of scientific research documents, whether they are published or not. The documents may come from teaching and research institutions in France or abroad, or from public or private research centers.
L'archive ouverte pluridisciplinaire HAL, est destinée au dépôt et à la diffusion de documents scientifiques de niveau recherche, publiés ou non, émanant des établissements d'enseignement et de recherche français ou étrangers, des laboratoires publics ou privés. 


\title{
Dynamic holographic memory showing readout, refreshing, and updating capabilities
}

\author{
S. Boj, G. Pauliat, and G. Roosen \\ Inśtitut d'Optique Théorique et Appliquée, Unité Associée au Centre National de la Recherche Scientifique, \\ Centre Scientifique d'Orsay, B.P. 147, 91403 Orsay Cedex, France
}

Received October 25, 1991

\begin{abstract}
We propose a new refreshing procedure for reading out a dynamic holographic memory without loss of information. The retrieved images are fed back to the memory after being thresholded and amplified. Experimental demonstration is performed with a $\mathrm{LiNbO}_{3}$ photorefractive crystal.
\end{abstract}

Multiplexing a large number of gratings in a volume holographic medium in such a way that all images stored can be addressed independently is important for a wide range of applications. ${ }^{1-3}$ In previous studies, large-capacity memories have been implemented by using photorefractive crystals. ${ }^{4,5}$ Moreover the newly demonstrated phase encoding of the reference beam technique, ${ }^{6,7}$ used with a ferroelectric spatial phase modulator, allows one to access the memory in the microsecond range. However, in dynamic materials such as photorefractive crystals the image retrieval partially erases the memory. Fixing techniques here are not adequate as they generally hinder further updating of the memory. In order to overcome this problem, a new architecture has been proposed recently that uses two holographic media. ${ }^{8}$ With the use of, for example, two photorefractive crystals, ${ }^{9}$ all the data stored in one of them are saved in the second crystal before the first crystal is read until there is complete erasure. The process is then repeated by exchanging the roles of the two crystals.

In this Letter we propose and demonstrate a more flexible readout and refreshing scheme that permits one to operate the memory for days without loss of information and with the use of one dynamic (write/erase) holographic medium only, here a single photorefractive crystal. The refreshing of the memory is obtained by adding a feedback loop to the photorefractive crystal. The images retrieved from the memory are fed back and rewritten inside the crystal after they are processed, thresholded, and amplified. As shown in Fig. 1(a), this process can be implemented by using an electronic loop. The images are, for example, detected by a CCD camera (with the shutter closed and the input beam off), processed by a host computer, and then sent back to the input spatial light modulator (SLM) and to the crystal by opening the shutter. For some applications an all-optical feedback loop can be preferred. All the pixels are processed in parallel by the optically addressed SLM (OASLM). The OASLM may work in a reflective geometry [Fig. 1(b)] or in a transmissive one. In this all-optical loop, the images to be written for the first time in the memory must also be written through the OASLM so that the relative phase between the image beam and its corresponding reference beam is the same for the first recording as for the refreshing procedure. Such a thresholding OASLM had already been inserted into a feedback loop by different authors, either without ${ }^{10}$ or with $^{9}$ a photorefractive crystal. These authors underlined the necessity of employing a spatial thresholding element in order to avoid walk-off of the images from their initial location. Because the characteristics of this all-optical loop strongly depend on the characteristics of the OASLM, we focus our attention here on the optoelectronic loop.

One operation cycle of the memory is illustrated by the kinetics of the amplitude $\delta n$ of the refractiveindex modulation of the $m$ th recorded hologram. This kinetics is represented in Fig. 2. This operation cycle can be broken up into three points:

(i) The length of time $T_{R}=T_{D}+T_{U}$ is the readout time during which the input shutter is closed. First the $m$ th image is retrieved during $T_{D}$, and then it is sent to the computer for processing. The processing time and the time required to write the processed image on the SLM are smaller than, or equal to, $T_{U}$. During this duration $T_{U}$, the memory can be used to retrieve all other desired images. Therefore, even if the computing time is important, it does not restrict the performances of the memory.

(ii) During $T_{W}$, the input shutter is opened and the $m$ th image is partially rewritten (refreshed) with the corresponding code (angle or phase) on the reference beam.

(iii) This refreshing procedure is then reproduced for the $(M-1)$ other images during $(M-1)$ $\left(T_{R}+T_{W}\right)$.

This readout and refreshing scheme is efficient even if $T_{W}$ is shorter than $T_{R}$. Indeed, even if the photorefractive writing $\tau_{w}$ and erasing $\tau_{e}$ time constants are equal, because the index modulation $\delta n_{\infty}$ of the recorded hologram (Fig. 2) is smaller than the maximum index modulation $\Delta n_{\max }$ that can be induced in the material, the writing speed $\left(\approx \Delta n_{\text {max }} / \tau_{w}\right)$ is much faster than the erasing one $\left(\approx \delta n_{\infty} / \tau_{e}\right)$.

When an all-optical loop is used, this readout and 

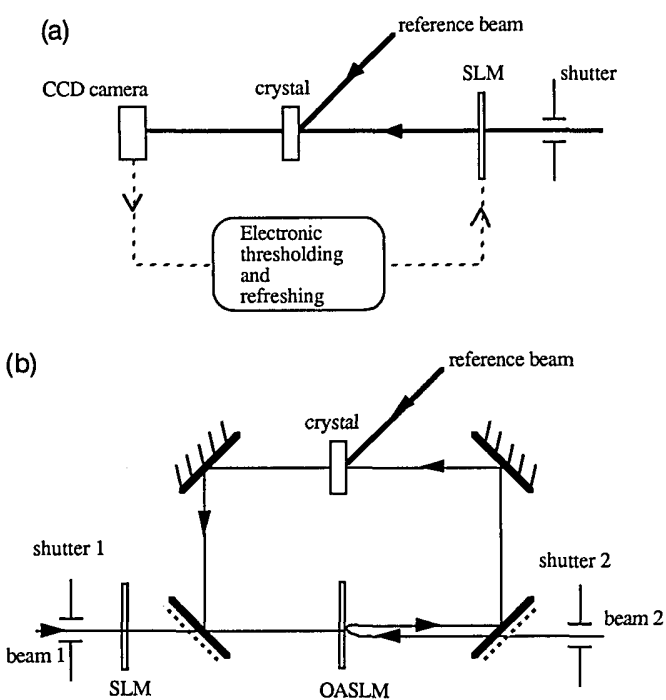

Fig. 1. (a) Schematic of a refreshing memory with an optoelectronic feedback loop. (b) Schematic of a memory with an all-optical feedback loop. The images are written on the OASLM either by input beam 1 from the electrically addressed SLM or by the retrieved image. The OASLM is read by auxiliary beam 2 .

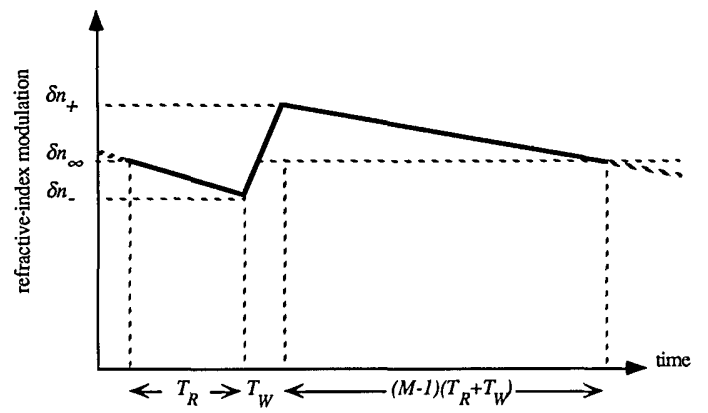

Fig. 2. Kinetics of the index modulation of the $m$ th stored image during one readout and refreshing cycle.

refreshing scheme can be slightly modified. For example, the time durations $T_{D}$ and $T_{W}$ may be combined. Updating of the memory can be performed with both electrical and optical loops by presenting new images during $T_{\mathrm{W}}$.

In order to give the main features of this refreshing scheme, we derive some simple expressions for the kinetics of the refractive-index modulation of the stored images. For the sake of clarity, we assume that the recording time constant and the erasure time constant are identical (equal to $\tau$ ) and are the same for all images. The calculation can be easily extended to the case in which these two time constants differ. Furthermore, in order to avoid unacceptably large temporal oscillations in the diffraction efficiency, we choose $T_{W}$ and $T_{R}$ so that $M\left(T_{R}+T_{W}\right) \ll \tau$. After a recursive calculation, provided that $M \gg 1$, we found for $\delta n_{\infty}$ and for the amplitude $C=\left(\delta n_{+}-\delta n_{-}\right) /\left(\delta n_{+}+\delta n_{-}\right)$of the temporal oscillations the following relations:

$$
C \approx \frac{M}{2} \frac{\left(T_{W}+T_{R}\right)}{\tau}, \quad \delta n_{\infty} \approx \frac{\Delta n_{\max }}{M\left(1+T_{R} / T_{W}\right)},
$$

where $\Delta n_{\max }$ represents the maximum index modulation that can be induced in the material. The first relation demonstrates that the amplitude $C$ of the oscillations can be kept at a reasonably low level provided that $M\left(T_{W}+T_{R}\right) \ll \tau$. The relation for $\delta n_{\infty}$ shows that our readout and refreshing procedure produces a slight decay of the refractive-index modulation compared with its maximum value $\Delta n_{\max } / M$ obtained for $T_{R}=0$. However, this decay can remain small; for example, it is only a factor of 2 for $T_{W}=T_{R}$.

We demonstrated the readout and refreshing procedure with the optoelectronic multiplexed holographic memory shown in Fig. 3. The writing wavelength was $514 \mathrm{~nm}$, and the holographic medium was a single $\mathrm{LiNbO}_{3}$ crystal with a $3-\mathrm{mm}$ thickness. The images, generated by a personal computer (PC), were impressed on the object beam by means of the liquid-crystal television screen (LCTV). This LCTV is imaged inside the crystal and then onto the CCD camera. The angular multiplexing technique was used for recording. Multiple holograms were stored by changing the incidence angle of a plane-wave reference beam. This was achieved in a traditional way, ${ }^{5}$ i.e., by rotating a galvanometer mirror (G.M.) placed at the object focal plane of the first lens of a refractive telescope while the crystal was at the image focal plane of the second lens. Each lens had a focal length of $92 \mathrm{~mm}$ and an aperture of $63 \mathrm{~mm}$. The mirror rotation was governed by the PC as a new image was displayed on the LCTV. The speed of this system, and thus the retrieval speed, is 300 scans (and therefore images) per second.

The images were recorded in the crystal by using the incremental procedure. ${ }^{6}$ For each position of the mirror, an input image was displayed on the LCTV and recorded for $8 \mathrm{~s}$ in the photorefractive crystal. Then a new mirror position and a new image were set for another 8-s recording of this new image. This procedure was applied to all 10 images that we wanted to record and continued for $20 \mathrm{cy}$ cles. As previously demonstrated, ${ }^{6}$ all images are stored with the same diffraction efficiency ( $1 \%$ here) in a time equal to the classical recording time of a single image, nearly $30 \mathrm{~min}$ for this crystal. This time will be reduced to less than $3 \mathrm{~min}$ with the $\mathrm{BaTiO}_{3}$ crystal and the power levels that we used previously. ${ }^{7}$ The recording speed limitation would

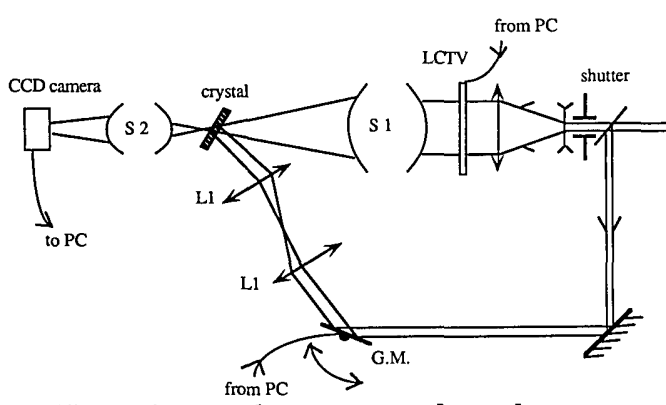

Fig. 3. Experimental setup used to demonstrate the readout and refreshing procedure. The scanning mirror G.M. is placed at the focus of the first lens L1 of the refractive telescope L1-L1, while the crystal is at the focus of the second lens. S1 and S2 are two optical systems that produce the image of the LCTV first in the crystal and then on the CCD camera. All the memory is driven by the personal computer PC. 
(a)
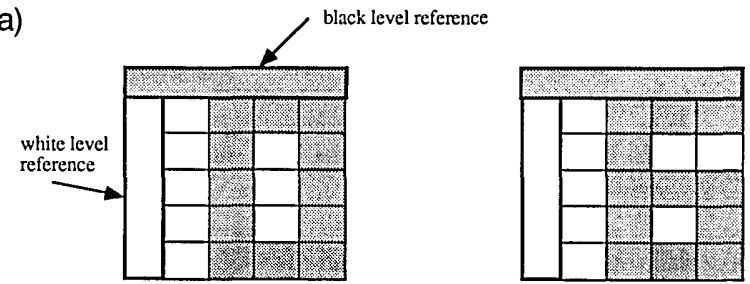

(b)
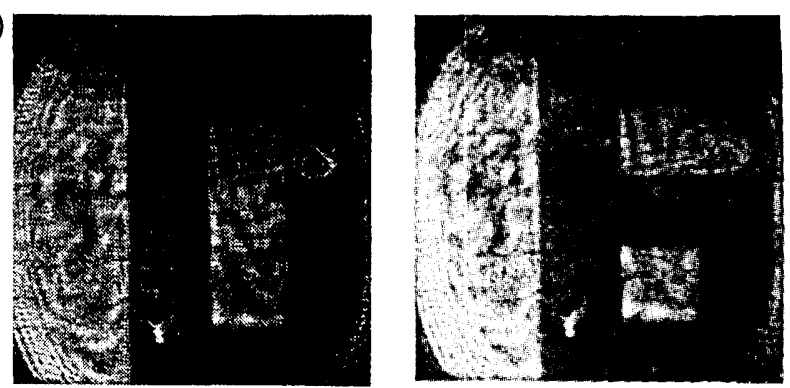

Fig. 4. (a) Two of the input images sent on the LCTV screen and (b) the corresponding retrieved images from the crystal, before the beginning of the readout and refreshing procedure. (a)

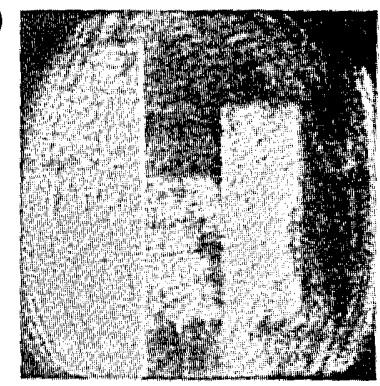

(b)

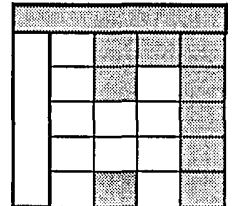

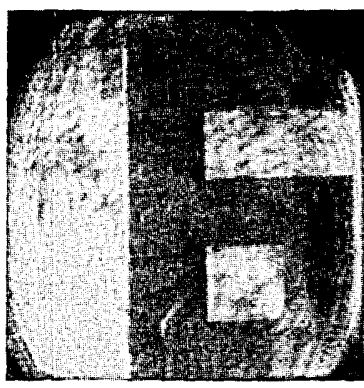

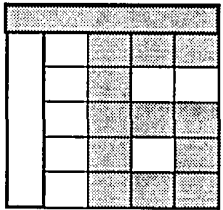

Fig. 5. (a) Same images as in Fig. 4 retrieved after $6 \mathrm{~h}$ of the readout and refreshing procedure and (b) the corresponding thresholded images: three pixels are missing in the left image.

thus come from the input SLM to be used in place of the LCTV. Indeed, if we consider the storage of 500 images with this incremental technique, the input images would have to be presented at a rate faster than $50 \mathrm{~s}^{-1}$. However, because the memory may be used during the time $T_{U}$ required to send the processed images to the SLM, the slowness of the SLM does not affect the retrieval speed.

Readout of one specific image was performed by closing the shutter and by rotating the mirror to the same position at which that image was recorded. Figure 4 shows 2 of the 10 images seen through the crystal presented for recording [Fig. 4(a)] and readout after recording the whole set of images [Fig. 4(b)].

To compensate for erasure that is due to image retrieval, we implemented the refreshing scheme with the optoelectronic feedback loop as described above. This demonstration was conducted with binary images only. Each image was generated with a black and white reference level in it before it was stored. At $t_{m}=0$ the shutter was closed, and until $t_{m}=T_{D}=25 \mathrm{~ms}$, the $m$ th image was retrieved and detected by the CCD camera. From $t_{m}=T_{D}$ to $t_{m}=T_{D}+T_{U}=8 \mathrm{~s}$, the intensity of each pixel was electronically compared with the value of the transmitted black and white levels and put back to either black or white. This time duration $T_{U} \approx 8 \mathrm{~s}$ may be employed to retrieve other images. For instance, with the scanning speed of our galvanometer mirror, as many as 2400 images can be retrieved during this time. An even larger number of images can be retrieved if a faster addressing system is used, such as the phase-encoding technique. This fast retrieval can be, for example, exploited to feed an optical correlator with the stored images as filters. At $t_{m}=T_{R}=T_{D}+T_{U}$, the shutter was opened for $T_{W}=8 \mathrm{~s}$, and the $m$ th image was rewritten in the crystal. This process was reproduced to refresh all other images. A more refined processing procedure would of course permit regeneration of gray-level images. Figure 5 shows the results. After $6 \mathrm{~h}$ of permanent reading out and simultaneous regeneration, most of the 10 images were still stored in the memory. Keeping the memory in the dark for the night, we continued the operation the next day. Without the regeneration loop, the images faded in approximately $30 \mathrm{~min}$. Note that some pixels are missing in some images. This is due to the low contrast of the LCTV and to spatial inhomogeneities in the transmission of the LCTV and in the diffraction efficiency. Some originally white (or black) pixels are therefore recognized as being black (or white) in the retrieved image and are finally reset to these new values in the holographic medium during the refreshing process. The use of a better SLM with a higher contrast may solve this problem.

We have proposed a new simple readout and refreshing procedure for holographic memory that uses either an optoelectronic feedback loop or an alloptical feedback loop. We have implemented this scheme with commercially available components.

\section{References}

1. P. J. Van Heerden, Appl. Opt. 2, 393 (1963).

2. A. Marrakchi, W. M. Hubbard, S. F. Habiby, and J. S. Patel, Opt. Eng. 29, 215 (1990).

3. D. Psaltis, D. Brady, and K. Wagner, Appl. Opt. 27, 1752 (1988).

4. D. L. Staebler, W. J. Burke, W. Phillips, and J. J. Amodei, Appl. Phys. Lett. 26, 182 (1975).

5. F. H. Mok, M. C. Tackitt, and H. M. Stoll, Opt. Lett. 16, 605 (1991).

6. Y. Taketomi, J. E. Ford, H. Sasaki, J. Ma, Y. Fainman, and S. H. Lee, Opt. Lett. 16, 1774 (1991).

7. C. Denz, G. Pauliat, G. Roosen, and T. Tschudi, Opt. Commun. 85, 171 (1991).

8. D. Brady, K. Hsu, and D. Psaltis, Opt. Lett. 15, 817 (1990).

9. H. Sasaki, Y. Fainman, J. E. Ford, Y. Taketomi, and S. H. Lee, Opt Lett. 16, 1874 (1991).

10. J. Taboury, J. M. Wang, and P. Chavel, in Proceedings of International Symposium on Optics in Computing (Centre d'Etudes et de Recherches de Toulouse, Toulouse, France, 1989), p. 77. 